\title{
HypoEnergy: Hybrid supercapacitor-battery power-supply optimization for Energy efficiency
}

\author{
Azalia Mirhoseini, Farinaz Koushanfar \\ Dept. of Electrical and Computer Engineering, Rice University, Houston, Texas \\ azalia@rice.edu,farinaz@rice.edu
}

\begin{abstract}
This paper presents HypoEnergy, a framework for extending the hybrid battery-supercapacitor power supply lifetime. HypoEnergy combines high energy density and reliable workload supportability of an electrochemical battery with high power density and high number of recharge cycles of supercapacitors. The lifetime optimizations consider nonlinear battery characteristics and supercapacitors' charging overhead. HypoEnergy-KI studies the hybrid supply lifetime optimization for a preemptively known workload and for one ideal supercapacitor. We show a mapping of HypoEnergy-KI to the multiplechoice knapsack problem and use dynamic programming to address the problem. HypoEnergy-KN considers the optimization for the known workload but in the case of having a nonideal supercapacitor bank that leaks energy. Evaluations on iPhone load measurements demonstrate the efficiency and applicability of the HypoEnergy framework in extending the system's lifetime.
\end{abstract}

\section{INTRODUCTION}

A portable system's lifetime and operability is severely constrained by its bounded power supply. Electrochemical batteries are the most widely used type of portable power supply in contemporary mobile and embedded systems. While the computational capacity has enjoyed an exponential growth over the past several decades, the battery capacity has only been linearly improving at a slow rate.

Some undesired properties of electrochemical batteries include their size limitations, bounded energy capacity and number of recharge cycles. Furthermore, chemical energy conversion has nonlinear properties, rendering battery's lifetime dependence on the incident load's current fluctuations and operational conditions.

Supercapacitors provide an alternative and emerging form of energy storage and delivery. The key benefits of them over the electrochemical battery include (i) orders of magnitude higher power density, (ii) orders of magnitude higher recharge cycle, and (iii) a much higher charge and discharge efficiency. However, while the energy density of supercapacitors is very high compared to traditional dielectric capacitors, it is lower than the electrochemical batteries and their leakage grows exponentially as the device is charged by a higher voltage.

A hybrid combination of batteries and supercapacitors can be used to exploit the best properties of both types of supplies [1]. Experiments on hardware platforms has shown the benefits of a hybrid power supply system with solar cells and supercapacitors in improving system's dynamic response and efficiency, in particular for pulsed load and transient loads with low duty cycles in sensor networks [2], [3], [4]. Experimental studies are of great value in demonstrating proof-of-concept and the need for more formal models and optimizations.

In this paper, we introduce Hybrid supercapacitor-battery power-supply optimization for Energy efficiency (HypoEnergy), optimization methods for improving a portable system's lifetime by a hybrid battery-supercapacitor power supply. We consider nonlinear battery models and supercapacitor characteristics. We first address the problem of optimizing the lifetime of a hybrid supply with a preemptively known load profile and an ideal supercapacitor with no leakage, referred to as HypoEnergy-KI. Next we address the hybrid source optimization problem for a preemptively known workload and a set of heterogeneous nonideal leaky supercapacitors, referred to as HypoEnergy-KN. Contributions are as follows:

- We formally define the Hypo-Energy-KI problem and demonstrate a mapping to the multiple-choice knapsack problem that can be solved by dynamic programming.

- We present a formal definition of Hypo-Energy-KN considering a supercapacitor set's nonideal characteristics.

- Extensive empirical measurements are used for modeling the nonideal supercapacitor leakage characteristics exploited in addressing the Hypo-Energy-KN problem.

- Experimental evaluations based on the iPhone load current profile shows the efficiency of the proposed methods in extending the portable system's lifetime.

\section{RELATED WORK}

Recent technological advances is promising a new generation of supercapacitors with improved energy density, power density, and leakage characteristics [5]. The improvements pave the way for widespread adaptation of supercapacitors in portable applications. Supercapacitors have been already adopted in high power commercial industrial applications and electronic vehicles for energy smoothing of high current loads.

A number of different models for characterizing nonlinear battery characteristics have been proposed [6], [7]. The nonlinear battery models were exploited in battery-aware optimization methods that aim at maximizing the battery's lifetime, including [8], [6], [9], [10]. The effectiveness of batteryaware optimization has been verified in theory, simulation, and measurements. A new aspect of our work is exploiting the nonlinear electrochemical battery characteristics for maximizing the hybrid battery-supercapacitor supply's lifetime for portable computing applications. 


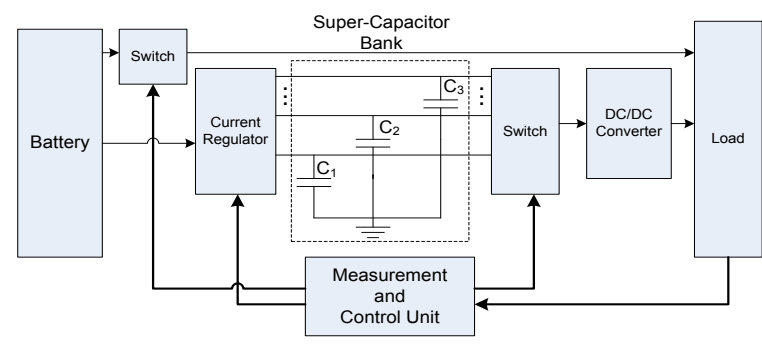

Fig. 1. Block diagram of HypoEnergy.

Using supercapacitors in conjunction with photovoltaic solar cells for charge storage is an active area of research and study, some recent papers include [2], [3], [4]. The analytical studies of this system and optimizations based on those studies rely on photovoltaic cell properties that are drastically different from conventional batteries. While the above mentioned systems have shown to serve sensor network application scenarios where the solar energy is available and loads commonly have very low duty cycles (the experiments are typically for the $1 \%-5 \%$ duty cycles) they are not suitable for many mobile application scenarios with higher workloads. Hybrid systems based on supercapacitors, batteries, or fuel cells were proposed [1], [11], [12], but exact models, detailed optimizations, or results for portable systems were not provided.

\section{HYPOENERGY OVERVIEW}

Rate capacity is the effect of nonlinear decreasing battery capacity, as the current drained out of it increases. Our goal in HypoEnergy is to consider the battery's rate capacity effect to maximize its lifetime. The higher power density supercapacitors are used to supply the loads with a high current demand. We aim at draining the minimum current out of the battery to charge the supercapacitor. Meanwhile, we consider a fast enough charging rate to meet the timing constraints.

Figure 1 shows the block diagram of HypoEnergy hardware. Based on the measurements of the load's power demand and the available energy and power in the hybrid source, the control unit decides how to serve the load considering battery's rate capacity effect. The control unit also decides the supercapacitors' charging policy (by the battery). The switches are to connect the selected energy source component to supply the load. A DC/DC converter is placed as an interface between the supercapacitor and the load to provide constant voltage. A current regulator is used to adjust the supercapacitor's charging current as decided by the control unit.

\section{HyPOENERGY-KI}

In this section, we consider a hybrid power source including a battery and a single ideal supercapacitor. The assumption is that the load power (current) demand is preemptively known. The objective is to optimize the power supply's lifetime. The hybrid energy supply system improves the battery lifetime by exploiting the supercapacitor's high power density to charge high current loads that exhaust the battery. Assuming that the load profile is known in advance, the objective is to find the optimal hybrid source operation. The method decides between using the battery or the supercapacitor to supply the load and also the charging policy for the supercapacitor. Let us more formally define the problem.

\section{Problem. Hypo-Energy-KI:}

Input. A battery power supply module $S_{0}$ with known discharge characteristics; a supercapacitor $S_{1}$ with capacity $C$ and the maximum achievable voltage $V_{\max }$; a set of tasks, each task $k$ is defined by the tuple $\left(\sigma_{k}, \Delta_{k}\right)$ denoting the task's charge demand and duration respectively for $1 \leq k \leq K$; and an integer $T_{B}$ denoting the deadline for performing all tasks.

Objective. Maximize the hybrid supply's lifetime.

We assume that the battery charge is enough to survive the task set's deadline, i.e., $\sum_{k=1}^{K} \Delta_{k} \leq T_{B}$. We set the total charge capacity of $S_{1}$ large enough to supply the task with the highest charge demand, i.e., $C V_{\max } \geq \max \left(\sigma_{k}\right)$ for $1 \leq$ $k \leq K$. To improve a battery lifetime given its characteristics, it is reasonable to assign tasks requiring a high current to the supercapacitor while the lower current tasks can be supplied by the battery. If the supercapacitor $S_{1}$ is selected to supply the load, one should ensure that the battery $S_{0}$ has charged $S_{1}$ at an earlier time. Given enough time, $S_{1}$ can integrate a lower current from $S_{0}$ and store the charge and then exploit its high power density to serve a high current load.

To solve the HypoEnergy-KI problem, assume that the battery can provide different current amplitudes for charging the supercapacitor. Since charging a supercapacitor at currents higher than the task's peak current would highly exhaust the battery, the upper bound current for charging the capacitor is set to the maximum task current. Without a loss of generality, suppose there are discrete number of charging current for supercapacitor denoted by $I_{m}^{c h}=\frac{m I_{M}^{c h}}{M}$ for $1 \leq m \leq M$. The durations needed to charge the supercapacitor to supply task $k$ is denoted by $\Delta_{k 1}^{c h}$ to $\Delta_{k M}^{c h}$, corresponding to the supercapacitor charging currents $I_{1}^{c h}$ to $I_{M}^{c h}$. Assuming an ideal supercapacitor, the charging duration can be obtained as:

$$
\Delta_{k m}^{c h}=\frac{\sigma_{k}}{I_{m}^{c h}} \quad 1 \leq m \leq M .
$$

We account for battery's rate capacity effect by assigning weights to the currents. The weights are selected such that they reflect the nonlinear battery lifetime with respect to its incident current. For finding the weight $w_{m}^{c h}$, we discharge the full battery with the constant current $I_{m}^{c h}$. Assuming that the discharging takes $T_{m}^{d}$ time, then the associated weight is $w_{m}^{c h}=\frac{1}{I_{m}^{c h} T_{m}^{d}}$. For weighting the tasks powered by the battery, the full battery is discharged with the current $\frac{\sigma_{k}}{\Delta_{k}}$ during the time interval $T_{k}$. Task $k$ 's weight is set to be $w_{k}=\frac{\Delta_{k}}{\sigma_{k} T_{k}}$. We define a set of auxiliary 0-1 integer variables $x_{k m}$ for $0 \leq m \leq M$ and $1 \leq k \leq K$ as follows:

i. $x_{k 0}$ : equals 1 if only the battery charges task $k$ with the total charge $\sigma_{k}$; equals 0 otherwise.

ii. $x_{k m}(1 \leq m \leq M)$ : equals 1 if only the supercapacitor charges task $k$ with the total charge $\sigma_{k}$ and the battery charges the supercapacitor with $I_{m}^{c h}$; equals 0 otherwise. 
The objective function is expressed below:

$$
\begin{array}{ll}
\text { OF : } & \min \Sigma_{k=1}^{K} \sigma_{k}\left(\Sigma_{m=1}^{M} x_{k m} w_{m}^{c h}+x_{k 0} w_{k}\right) . \\
C^{\prime} s: & \sum_{k=1}^{K}\left(\Sigma_{m=1}^{M}\left(x_{k m} \Delta_{k m}\right)+\Delta_{k}\right) \leq T_{B}, \\
& \sum_{m=0}^{M} x_{k m}=1 \quad 1 \leq k \leq K .
\end{array}
$$

The objective function aims at minimizing the weighted charge consumptions. The first constraint assures that all tasks are done within the time budget $T_{B}$. The second constraint ensures that at least one supply option is used for each task $k$.

Optimization 2 is in form of the well-known multiplechoice knapsack problem. Since the supply scheduling is preemptively computed, we opt to use a dynamic programming algorithm adopted from Dudzinski and Walukiewicz [1987] to optimally address the problem.

\section{HYPOENERGY-KN}

In this section, we consider a hybrid source with a battery and multiple non-ideal supercapacitors (a supercapacitor bank) that leak energy. Given the solution of HypoEnergy-KI, we would know whether the supercapacitor or the battery is in charge to supply a task. If the former case is true, we would also know at what current the supercapacitor should be charged by the battery to have sufficient charge for the task. In HypoEnergy-KN, we have a bank of supercapacitors that can be beneficial in two ways. One is pipelining the power source, i.e., the battery can charge a supercapacitor from the bank while another supercapacitor is supplying the load. The second benefit is that a heterogenous supercapacitor bank would give the flexibility to choose the best supercapacitors to charge or discharge so as to minimize the leakage for each of the tasks. The HypoEnergy-KN problem is expressed as follows:

\section{Problem. HypoEnergy-KN:}

Input. A heterogenous supercapacitor bank with elements $S_{i}$ and corresponding capacities $C_{i}$ for $1 \leq i \leq N$ with known leakage characteristics; Solution of HypoEnergy-KI, i.e., $x_{k m}$ for $1 \leq k \leq K$ and $0 \leq m \leq M$.

Objective. Minimize the supercapacitor bank's leaked energy by choosing the best supercapacitors to charge or discharge.

One notable limitation of a supercapacitor is its leakage. Figure 2(a) shows the dependency of leakage to the supercapacitor's voltage for different capacities. Charging a supercapacitor would increase its voltage and leakage power. Selecting the best supercapacitors to charge or discharge is the goal of HypoEnergy-KN.

Assume that there are three supercapacitors with $200 \mathrm{~F}, 400 \mathrm{~F}$ and 2000F capacities. The leakage profiles are adopted from [4]. At each sampling time, we decide to fill the supercapacitors with a charge value of $\sigma=20 \mathrm{C}$. The increment in the three supercapacitor's leakage power is plotted versus their voltages in Figure 2(b). We see that both the SoC and the capacity are important from the leakage perspective. For example, if all the supercapacitors are initially at $2 \mathrm{~V}$, their leakage powers barely increase after being charged. Thus, supercapacitor selection does not affect the leakage power. However, if the supercapacitors were initially at $2.5 \mathrm{~V}$, selecting the $2000 \mathrm{~F}$ supercapacitor to charge can be justified, since then the leakage power would increase by less than $5 \mathrm{~mW}$. This is much less than the leakage increment if we had selected any of the other two capacities. Therefore, for solving the problem of charging the supercapacitor bank to a given amount with a minimal leakage increment, both the SoC and the supercapacitors' capacity should be considered. For each of the tasks $\left(\sigma_{k}, \Delta_{k}\right)$ that are selected (from equation 2) to be supplied by the supercapacitors, we formally define the problem of minimizing the supercapacitors leaked energy:

$$
\begin{array}{ll}
\text { OF }: & \min \Sigma_{i=1}^{N}\left(\int_{\sigma_{i}}^{\sigma_{i}+\Delta \sigma_{c_{i}}} P_{L}^{i} d t+\int_{\sigma_{i}+\Delta \sigma_{c_{i}}}^{\sigma_{i}} P_{L}^{i} d t\right) .(3) \\
C^{\prime} s: & \Sigma_{i=1}^{N} \Delta \sigma_{c_{i}}=\sigma_{k} .
\end{array}
$$

Where $P_{L}^{i}$ is the leakage power of $C_{i}$ and the unknown $\Delta \sigma_{c_{i}}$ is the portion of $\sigma_{k}$ delivered to $C_{i}$, for $1 \leq i \leq$ $N$. The bounds of the integrals, are the charge values of $C_{i}$ at the beginning/end of the charging/discharging interval assuming that their initial charge is $\sigma_{i}$. While charging (first integral), the time and charge deviations are related as follows, $d t=\frac{d \sigma}{I}=\frac{C_{i} d v}{I_{m}^{c h}}$. While discharging (second interval), $d t=\frac{d \sigma}{I}=\frac{C_{i} d v}{\left(-\frac{\sigma_{k}}{\Delta_{k}}\right)}$. It is assumed that the supercapacitors are fully discharged in the beginning. In order to solve Equation 3, leakage power $P_{L}$ should be characterized. We used curve fitting techniques on the measured data for estimating $P_{L}$ as a function of capacitance and voltage of the supercapacitor. Our results showed that an exponential fit gives a very accurate model with the least number of model parameters. Previous work has considered piecewise linear or polynomial fits that use considerably more parameters for the same accuracy [4], [3]. Our exponential fit is shown as $P_{L}\left(C_{i}, v\right)=\alpha_{i} e^{\beta_{i} v}(m W)$. Substituting the leakage model in Optimization 3, we see that the objective function is nonconvex (or non-concave) and non-smooth. Therefore, finding the optimum solution adaptively is a challenge. To overcome this hurdle, in HypoEnergy-KN, we consider filling only one supercapacitor with $\sigma_{k}$. Other than computational complexity, another reason for charging the supercapacitors one at a time, is the technical difficulties arising from charging them with different voltage stages in parallel. To find the optimal supercapacitor to charge/discharge we use the following objective: $C_{\text {opt }}\left(\sigma_{k}\right)=\operatorname{argmin}_{(1 \leq i \leq N)}\left(\int_{\sigma_{i}}^{\sigma_{i}+\sigma_{k}} P_{L}^{i} d_{t}+\int_{\sigma_{i}+\sigma_{k}}^{\sigma_{i}} P_{L}^{i} d_{t}\right)$. Note that $C_{\text {opt }}$ should be large enough to be able to store $\sigma_{k}$, i.e., $C_{\text {opt }} V_{\max } \geq \sigma_{k}$.

\section{EXPERIMENTAL RESULTS}

In this section, we evaluate the effectiveness of the HypoEnergy framework in extending the portable supply system lifetime. The evaluations are performed on real measurements of iPhone taken at our department [13]. The battery current measurements for different loads are averaged over a period of one minute rounded by $10 \mathrm{~mA}$. Based on the measurements, we constructed five benchmark load task sets for five users denoted by $U \operatorname{ser}_{1}$ to $\operatorname{User}_{5}$. The measured current values for 


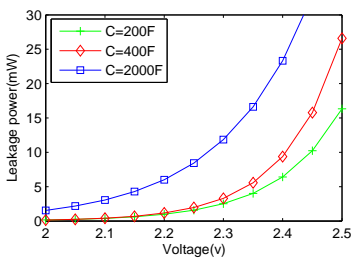

(a) Leakage power as a function of (b) Increment in leakage for a fixed voltage for different capacities.

added charge.

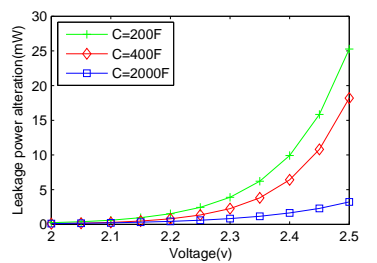

Fig. 2.

\begin{tabular}{|l|l|l|}
\hline Profile & Description & $\mathbf{I}(\boldsymbol{m A})$ \\
\hline$P 1$ & display off, airplane mode & 40 \\
\hline$P 2$ & display off, default mode & 100 \\
\hline$P 3$ & web browsing over 3G & 310 \\
\hline$P 4$ & tap-tap revenge game & 220 \\
\hline$P 5$ & GTI racing 3D game & 400 \\
\hline$P 6$ & youtube over WiFi & 260 \\
\hline$P 7$ & youtube over 3G & 350 \\
\hline$P 8$ & talking on the phone & 170 \\
\hline
\end{tabular}

Fig. 3. iPhone current profiles.

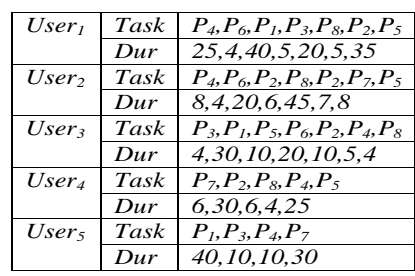

Fig. 4. Users' workload profiles. the iPhone profiles and the user task sets and corresponding durations are shown in Figures 3 and 4 respectively. The metric we used for evaluating improvement in battery lifetime is charge saving factor. The metric quantifies the percentage of charge saved by HypoEnergy to the total load's charge demand. For computing the charge consumption of the battery, the rate capacity effect is taken into account by exploiting the current weights similar to Section IV. The charge consumption is directly related to the lifetime extension.

For the HypoEnergy-KI case, user task sets are known in advance. In our simulations, we use a Li-Ion battery with $40000 \mathrm{~mA}$-min capacity and the voltage of $4.3 \mathrm{~V}$. A $400 \mathrm{~F}$ supercapacitor is used to ensure that it can supply the highest charge demand for the user tasks which is $p_{5}(400 \mathrm{~mA})$ for 35 minutes by user $_{1}$. In HypoEnergy-KN, the same battery is used with a supercapacitor bank that includes a $50 F$, a $200 F$ and a $400 F$ supercapacitor. The fitting coefficients $\alpha_{i}$ and $\beta_{i}$ (introduced in Section V) for the three supercapacitors are:

\begin{tabular}{rrr}
\hline $\operatorname{Capacity}(F)$ & $\alpha$ & $\beta$ \\
\hline 50 & $4.05 \times 10^{-9}$ & 8.52 \\
200 & $1.14 \times 10^{-9}$ & 9.354 \\
400 & $1.26 \times 10^{-10}$ & 10.43 \\
\hline
\end{tabular}

Table I shows the charge efficiency of HypoEnergy-KI. We examine the charge saving factor for different time budgets for the users. $T_{B}(\mathrm{~min})$ is the total duration of each user's task set. As the table shows, increasing the time budget extends the savings by allowing the battery to charge the supercapacitor at a lower pace. Also, we see that for a user with a higher load current, the metric improvement is more significant.

Table II shows the charge saving factor in HypoEnergyKN with considering the leaky supercapacitors. The results show that in the worst case, the leaked charge would reduce the efficiency up to $4.5 \%$ in comparison to the ideal case (HypoEnergy-KI). As the time budget $T_{B}$ increases, the leakage increases slightly (less than $0.4 \%$ ).
TABLE I

THE CHARGE SAVING FACTOR IN HYPOENERGY-KI.

\begin{tabular}{rrrrrr}
\hline & $T_{B}+10$ & $T_{B}+20$ & $T_{B}+30$ & $T_{B}+40$ & $T_{B}+50$ \\
\hline User $_{1}$ & 12.3 & 13.9 & 15.5 & 16.7 & 17.6 \\
ser $_{2}$ & 9.3 & 11.0 & 11.4 & 12.5 & 13.5 \\
Sser $_{3}$ & 12.7 & 13.6 & 15.2 & 16.6 & 17.8 \\
ser $_{4}$ & 15.7 & 17.5 & 20.0 & 22.0 & 23.4 \\
User $_{5}$ & 12.9 & 15.1 & 16.7 & 18.0 & 19.2 \\
\hline
\end{tabular}

TABLE II

THE CHARGE SAVING FACTOR IN HYPOENERGY-KN.

\begin{tabular}{rrrrrr}
\hline & $T_{B}+10$ & $T_{B}+20$ & $T_{B}+30$ & $T_{B}+40$ & $T_{B}+50$ \\
\hline User $_{1}$ & 8.1 & 9.7 & 11.3 & 12.3 & 13.1 \\
User $_{2}$ & 8.4 & 10.0 & 10.4 & 11.5 & 12.5 \\
User $_{3}$ & 11.6 & 12.5 & 14.1 & 15.4 & 16.5 \\
User $_{4}$ & 13.9 & 16.7 & 18.2 & 20.1 & 21.1 \\
User $_{5}$ & 11.4 & 13.6 & 14.2 & 16.4 & 17.6 \\
\hline
\end{tabular}

\section{CONCLUSiON}

This paper introduced HypoEnergy, a framework for extending portable system's lifetime using a hybrid batterysupercapacitor power supply. HypoEnergy-KI aimed at prolonging the lifetime for preemptively known load and ideal supercapacitors. HypoEnergy-KI was solved by a mapping from the multiple-choice knapsack problem and a dynamic program for solving this problem. HypoEnergy-KN objective was to decrease the leaked energy for a bank of nonideal supercapacitors. Evaluating the methods on iPhone workload power profiles demonstrated the effectiveness of HypoEnergy in improving system's lifetime.

\section{REFERENCES}

[1] F. Koushanfar, "Hierarchical hybrid power supply networks," in $D A C$, 2010, pp. 629-630.

[2] D. Brunelli, C. Moser, L. Thiele, and L. Benini, "Design of a solarharvesting circuit for batteryless embedded systems," TCAS I: Regular Papers, vol. 56, no. 11, pp. 2519-2528, 2009.

[3] G. Merrett, A. Weddell, A. Lewis, N. Harris, B. Al-Hashimi, and N. White, "An empirical energy model for supercapacitor powered wireless sensor nodes," in ICCCN, 2008, pp. 1-6.

[4] T. Zhu, Z. Zhong, Y. Gu, T. He, and Z. Zhang, "Leakage-aware energy synchronization for wireless sensor networks," in MobiSys, 2009, pp. 319-332.

[5] M. Stoller, S. Park, Y. Zhu, J. An, and R. Ruoff, "Graphene-based ultracapacitors," Nano Letters, vol. 8, no. 10, pp. 3498-3502, 2008.

[6] D. Rakhmatov and S. Vrudhula, "Energy management for batterypowered embedded systems," TECS, vol. 2, no. 3, pp. 277-324, 2003.

[7] C. Park, J. Liu, and P. Chou, "B\#: A battery emulator and power profiling instrument," IEEE $D \& T$, vol. 18, no. 2, pp. 150-159, 2005.

[8] L. Benini, G. Castelli, A. Macii, and R. Scarsi, "Battery-driven dynamic power management," IEEE D \& T, vol. 18, no. 2, pp. 53-60, 2001.

[9] S. Choi, H. Cha, and R. Ha, "A selective dvs technique based on battery residual," Microproc. and Microsys., vol. 30, no. 1, pp. 33-42, 2006.

[10] Y. Cai, M. Schmitz, B. Al-Hashimi, and S. Reddy, "Workload-aheaddriven online energy minimization techniques for battery-powered embedded systems with time-constraints," TODAES, vol. 12, no. 1, pp. $1-24,2007$.

[11] T. Yalcinoza and M. Alam, "Improved dynamic performance of hybrid PEM fuel cells and ultracapacitors for portable applications," International Journal of Hydrogen Energy, vol. 33, no. 7, pp. 1932-1940, 2008.

[12] M. Pedram, N. Chang, Y. Kim, and Y. Wang, "Hybrid electrical energy storage systems," in ISLPED, 2010, pp. 363-368.

[13] C. Shepard, C. A. Rahmati, Tossel, L. Zhong, and P. Kortum, "Livelab: measuring wireless networks and smartphone users in the field," HotMetrics, 2010. 